\title{
Competition Model and Contrastive Lexical Competition
}

\author{
Meisam Ziafar ${ }^{1}$, Ehsan Namaziandost ${ }^{2}$
}

\author{
${ }^{1}$ Assistant Professor, Department of English Language Teaching, Ahvaz Branch, Islamic Azad University, Ahvaz, Iran \\ meisam-ziafar@iauahvaz.ac.ir \\ ${ }^{2} \mathrm{Ph} . \mathrm{D}$. Candidate in TEFL, Department of English, Faculty of Humanities, Shahrekord Branch, Islamic AzadUniversity, Shahrekord, Iran \\ e.namazi75@yahoo.com
}

\begin{abstract}
The Competition Model (CM) embraces lexicalist and functionalist approach to language structure and function. What is highly emphasized in this model is a lexicalist functionalism through which syntactic patterns are directed and controlled by lexical items. CM tenets resemble to that of Haliday's systemic-Functional linguistics in that it only deals with form-meaning relations within a text and not in the real world. A new Competition Model needs to be introduced which is more pragmatic-oriented through taking formulaic sequences as forms to be mapped onto real world pragmatic functions. CM must free itself from the mere focus on sentence processing studies and involve itself with more pragmatic manifestations of form-function relations. It is claimed that within the models in which there is an architecture that utilizes lexical categories to build "valence bridges", L1-L2 translation equivalents facilitate crossing valence bridges which helps in discovering forthcoming elements and filling syntactic slots.
\end{abstract}

Keywords_Lexical Competition, Competition Model.

\section{Competition Model and Contrastive Lexical Competition}

According to MacWhinney, Bates, and Kleigl (1984) the Competition Model (CM) is based on the idea that"the forms of natural languages are created, governed, constrained, acquired and used in the service of communicative functions." (p. 128)

According to Jordan (2004) the Competition Model (CM) proposed by Bates and MacWhinneyin 1982 challenges two approaches to language: innateness and formalism. Despite Chomsky's Principles and Parameters Model CM considers language as 'non-modular' and 'nonspecific', on the other hand it is believed that language learning comes about due to similar 'cognitive mechanisms' to those effective in other types of learning. Jordan (2004) argues that $\mathrm{CM}$ also challenges Chomskyian formalism through contending that language form and function are not separable. Jordan (2004) argues that as a result of challenging both innateness and formalism, CM demonstrates another contrast to Chomsky's UG theory, which is based on the fact that when Chomsky's UG is a theory about competence, Bates and MacWhinney's CM puts forward a theory of performance and is emergentist in its assumptions (MacWhinney, 2006a).

Jordan (2004) maintains that the CM pledge allegiance to four major theories:

1- Lexical functionalism: According to Functionalism the forms of language are dictated by the communicative functions they conduct; language represents an array of mappings between forms and functions. MacWhinney (2004b) places importance on the role of a lexical approach by asserting that $\mathrm{CM}$ is being developed through principles of lexicalist, bottom-up learning.

2- Connectionism: CM takes advantage of connectionist models in mapping interactions between lexical mappings. Connectionism is in sharp contrast with nativists' contention that brain functions as a 'symbol processing device' similar to that of a digital computer. Connectionism believes that the brain employs patterns of connectivity and activation in its computations. According to Kempe and Mac Whinney (1998) connectionist theories and the CM are all in the same boat in their claim that the concept of rule should be dispensed with and that learning takes place through gradual strengthening of the association between 
co-occurring elements of the language. However, the CM is different from the Network model and other connectionist models in that when such models emphasize the structure of lexicon and the 'intralexical' relations affecting learning and representing inflectional paradigms, the $\mathrm{CM}$ focuses on the inflections as cues to underlying thematic roles and pragmatic functions.

3- Input-driven learning: Language learning can be explained according to input rather than innate principles and parameters. The key construction in this explanation is cue validity. MacWhinney (1997, cited in Jordan, 2004) introduces cue validity as a conditional probability:

Ellis (2006, p. 169) defines the role of cue validity in the Competition Model as follow:

The Competition Model explains the transition of cue use from overall validity to conflict validity with a learning-onerror mechanism. A strength counter is maintained for each cue, and in deciding a role, the noun with the largest total cue strength is assigned to that role. When a role is assigned incorrectly, cues that could have predicted the correct answer have their strength increased. There is no increase in strengths in the case of a correct assignment. Initially, all cue strengths are small random values, so errors are made over a representative sampling of all sentences. Cue strengths are thus incremented proportionally to the ability of the cue to predict correct assignment over all sentences (overall validity). Errors continue to decrease, and at some point, sentences that do not have cues conflicting in the prediction of assignment do not produce errors. Then, cue strengths are incremented for sentences with conflicting cues (conflict validity).

4- Capacity: Short-term verbal memory is limited in capacity and utilizing language in real time is constantly affected by these limitations.

In brief, the Competition Model argues that language encodes functions like 'topic' and 'agent' onto surface grammatical conventions in various ways such as word order and subject-verb agreement. Because of the limits on processing, these functional categories compete for control of the surface grammatical conventions. Speakers of languages use four types of cues - word order, vocabulary, morphology, and intonation-to facilitate their interpretation of these form-function mappings. Because of the principle of limited capacity mentioned above, human languages find different ways of using these cues. (Jordan, 2004, p. 237)

According to MacWhinney (1997, cited in Jordan, 2004) the CM believes that the process of second language learning begins with a 'parasitic lexicon', a 'parasitic phonology', and a 'parasitic set of grammatical constructs'. Through time the second language emerges out of this parasitic status and turns into a complete language per se.

Matessa and Anderson (2000) define CM as follow:

The Competition Model (Bates \& MacWhinney, 1989) can explain the transition of cue use from overall validity to conflict validity with a learning-on-error mechanism. In the model, a strength counter is maintained for each cue, and in deciding a role, the noun with the largest total cue strength is assigned to that role. When a role is assigned incorrectly, cues that could have predicted the correct answer have their strength increased. There is no increase in strengths in the case of a correct assignment. Initially, all cue strengths are small random values, so errors will be made over a representative sampling of all sentences. Therefore, cue strengths are incremented proportionally to the ability of the cue to predict correct assignment over all sentences (overall validity). Errors continue to decrease, and at some point, sentences that do not have cues conflicting in the prediction of assignment do not produce errors. Then, cue strengths are incremented for sentences with conflicting cues (conflict validity). (p. 264)

MacWhinney and Pleh (2000) define the CM as follow:

The Competition Model adopts a lexicalist, functionalist approach to language structure and function (MacWhinney, 1997). It relies on connectionist modelling and parameterised mathematical modelling as tools to account for input-driven learning during language acquisition and cue interaction during processing. (p. 70)

Behrens (2009) maintains that each language and dialect possesses its own affordances regarding the cues it provides for learning its structure. Behrens (2009) contends that according to MacWhinney and Bates (1987) such cues are in competition with each other to the extent that the ease or difficulty of acquisition can be predicted through cue validity, which is a function of its availability and reliability, in relation to the cost of processing the cue. Cue cost is involved in computing the difficulty of processing. More frequent elements are more easily detected and stored compared to less frequent elements (Behrens, 2009).

In regard to predicting the order of acquisition in different languages, Behrens (2009) puts emphasis on the role of the $\mathrm{CM}$ as a factor in such predictions through allowing us to compute the impact of the relation between different cues 
inacquisition.He contends that the same prediction efforts are done through probabilistic theories of language which "have been applied to the acquisition of part-of-speech categories through observing co-occurrence statistics or through multiple-cue integration, i.e. prosodic, phonological and distributional cues." (Behrens, 2009, p. 213)

MacWhinney, Taraban, \& McDonald (1989) maintain that according to $\mathrm{CM}$ 'cue acquisition' and 'cue strength' are dependent on four major cue properties: detectability, task frequency, availability, and reliability. For a child to learn using cue, the child must first be able to detect the presence of a cue. Considering frequency it can be claimed that the more often a child is required to process a particular type of cue, or assign a certain category, the sooner it will be possible for him/her to master a cuecategory relationship. All other things being equal, the more a marker is available, the sooner it is acquired. Finally reliability is defined as the number of times a cue is associated with a certain category, such as masculine, feminine, and neutral, at the presence of the cue.

\section{Lexical Functionalism}

Ellis (1999) emphasizes the role of lexical functionalism in the $\mathrm{CM}$ and maintains that in $\mathrm{CM}$ syntactic patterns are controlled and governed by lexical items. $\mathrm{He}$ further maintains that recent studies in CM are simulating language performance by means of simple connectionist models through relating lexical cues like word order, verb agreement morphology, case marking, etc., to functional interpretations like agency, topicality, perspective, etc., in sentence comprehension and production. He maintains that $\mathrm{CM}$ is also involved in prediction of transfer, a process which serves the good job of reviving contrastive analysis in a new probabilistic guise.

In regard to lexical control of processing MacWhinney (1994)tries to extend the construction of a connectionist basis of CM through employing lexical networks in order to control syntactic processing in both comprehension and production. The main factor in this model is an architecture that utilizes lexical categories to build "valence bridges". He defines valence bridges as "semantic-syntactic connections between slots activated by lexical items and the arguments that fill these slots" (MacWhinney, 1994, p. 21).

For comprehension the model would look like this:

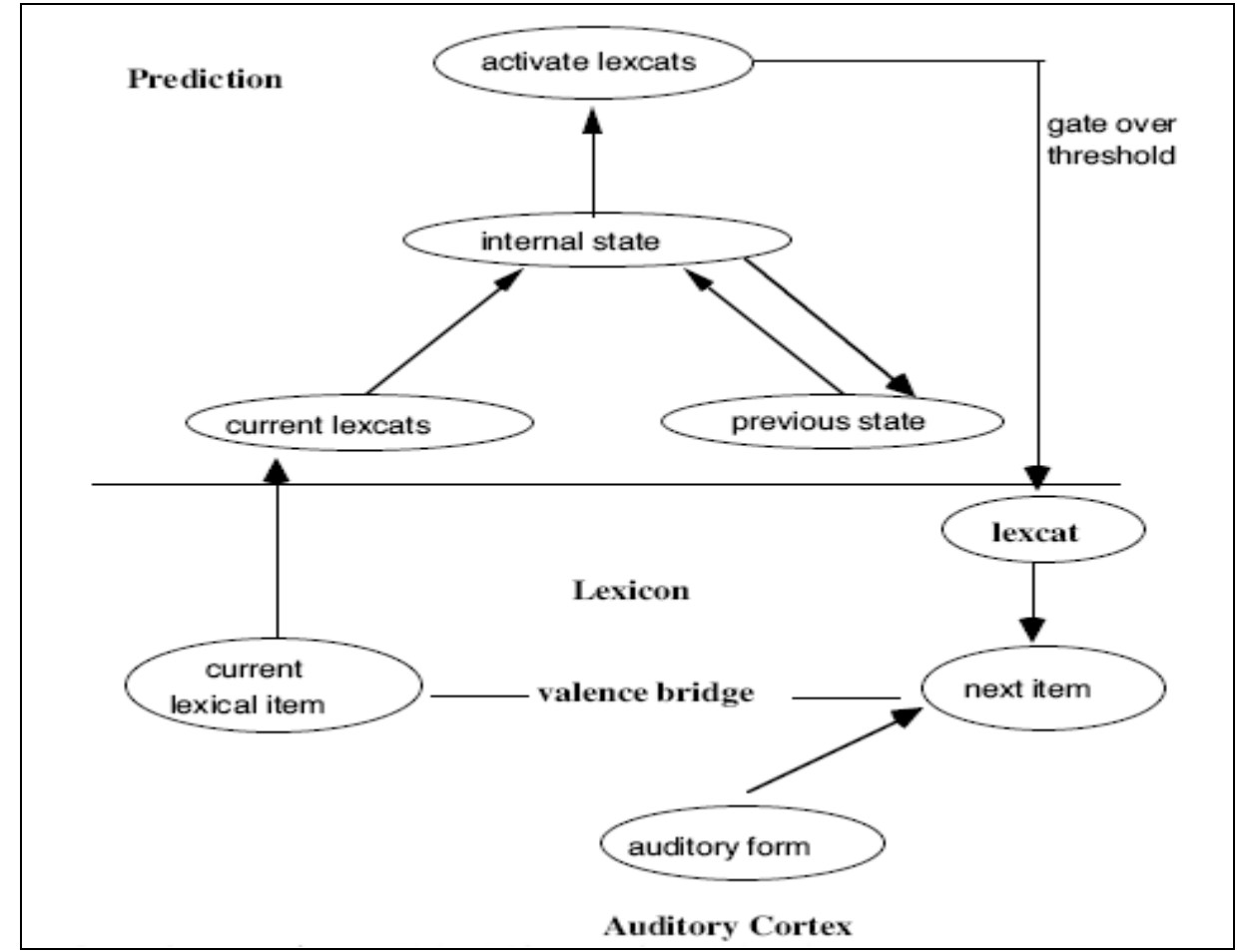

Fig.1: Taken from MacWhinney, 1994, p. 22 
For production the model would look like this:

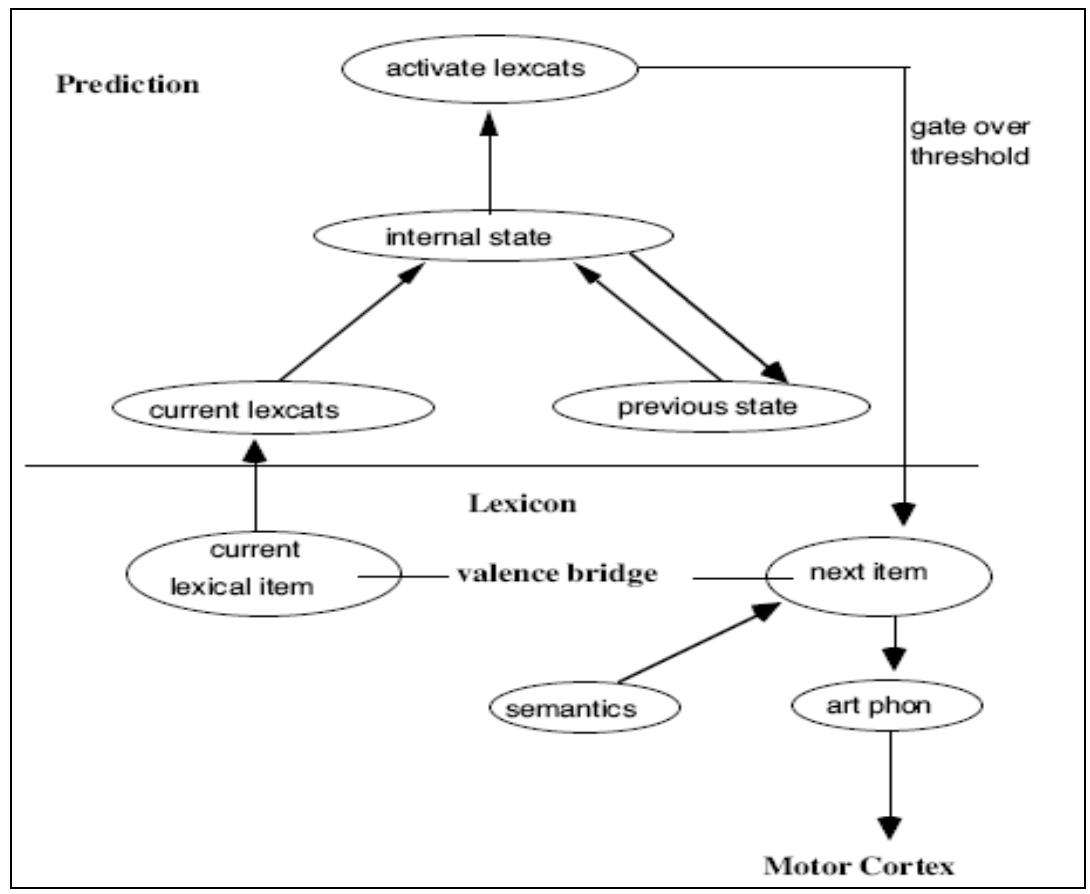

Fig.2: Taken from MacWhinney, 1994, p. 24

According to MacWhinney, Bates, and Kleigl (1984) the six fairly strong claims set forth by the Competition Model (CM) about the control of sentence processing are as follow.

1- Direct mapping: there are only two defined levels of processing in the model: a functional level (through which all meanings and intentions expressible in an utterance are introduced) and a formal level (through which the surface forms proper for a certain meaning/intention configuration are introduced). It is argued that the mappings between the formal and the functional levels are direct.

2- Multiplicity of form-function mappings: it is believed that the mapping of one form onto one function is quite rare in natural languages. Through polysemy for example a certain form may map onto several functions and a certain function may map over several forms.

3- Coalitions and breakdowns of coalitions: is based on the idea that the mappings between forms and functions are not selected by chance, rather it is claimed that certain things tend to go together naturally.As an example, the functions of agent, actor, and topic prototypically map onto the set of devices that constitute 'subject'. In this case it is said that a coalition of functions in mapped onto a coalition of forms.
4- Competition: the model holds that there is a dynamic control of mapping of form onto function in comprehension and of function onto form in production.

5- Cue strength: it is believed that in the set of many-tomany mappings each connection between a form and a function is assigned a weight or strength. Such theory strength has been used to justify and description of transitional stages in language acquisition. It is believed that contrary to more deterministic models of language change in which it is contended that rules are whether present or absent, the CM allows apparent rules or conventions to emerge gradually, through a constant increase in the determining force of probabilistic form-function mappings. 6- Cue validity: it is maintained that the weights of cues in one language are reflections of their relative cue validity in that language. Based on this assumption it is possible to attend to a set of form-function mappings estimate their relative cue validity and then employ these estimates in order to predict both order of acquisition and relative usage in adult processing. Cues with the most relaiability and applicability are the most valid cues. Cues with low applicability and high reliability are fairly high in validity, although it is not possible to trust them as much as cues that are always present when needed. Finally cues with low reliability are the least valid, even though when more valid 
cues are not at hand, even unreliable cues are worthy to be attended to.

In their article MacWhinney, Pleh, and Bates (1985) add a seventh component to the claims in CM which is called ongoing updating.

7- Ongoing updating:for the purpose of controlling the interaction various cues that affect sentence processingit is believed that the parsing system is involved in an ongoing updating of relating nouns to case roles. The authors provide us with an example. In the case of the sentence "the dogs are chasing the cat" the allocation of "dog" as the agent is at first aided by its placement in the initial position. In the second place, since "the dogs" agrees with "are chasing" in number further supports this allocation. Finally, the appearance of "cat" postverbally its binding to the object case-role further supports the 'candidacy' of "dog" as the agent. Thus, the candidacy of "dog" is updated at each stage of sentence processing. Marcus (1980, cited in MacWhinney, Pleh, \& Bates,1985) contend that since the language designs the cues to support ongoing updating the need for backtracking is minimized. Harrington and Dennis (2002) also maintains that the CM framework has proven the fact that learners' speed in learning and processing a given form-function relationship represents the frequency of different cues in the language. Cues such as word order, case making, and animacy can be correlated to represent agency (MacWhinney, cited in Harrington\& Dennis, 2002).

According to Dopke (2000) the CM believes that
He maintains that grammar is acquired through constructing connections between meaning and form based on the structural cues and by means of the completion of cues for related functions. He contends that the successful determination ofsuch competition leads to robust structural schemata. Cues with higher reliability, frequency, and availability and salience overcomeless strong cues. As a result, cues with higher strength, are more quickly allocated to their proper grammatical functions compared to weak cues. If competition happens between several cues for the same function, or if the same cue stands for several functions, the acquisition of a certain structure will be postponed. Such tensions between similarities and contrasts drive the syntax acquisition.

Ellis (2002) maintains that the CM is based on lexical functionalism through which syntactic patterns are directed and controlled by lexical items. Lexical items are responsible for providing cues for functional interpretations in sentence comprehension or production. Some cues have more reliability than others. The language learners' duty is to discover which cues are the most valid predictors. The $\mathrm{CM}$ represents the paradigmatic example of 'constraintsatisfaction' statements of language comprehension.

MacWhinney (2005) introduces the Unified Competition Model in which it is assumed that L1learning mechanism is a subset of L2 learning and bilingualism mechanism. The components of such a unified model are shown in the following figure (Figure 3).

language processing takes place at the surface of utterances.

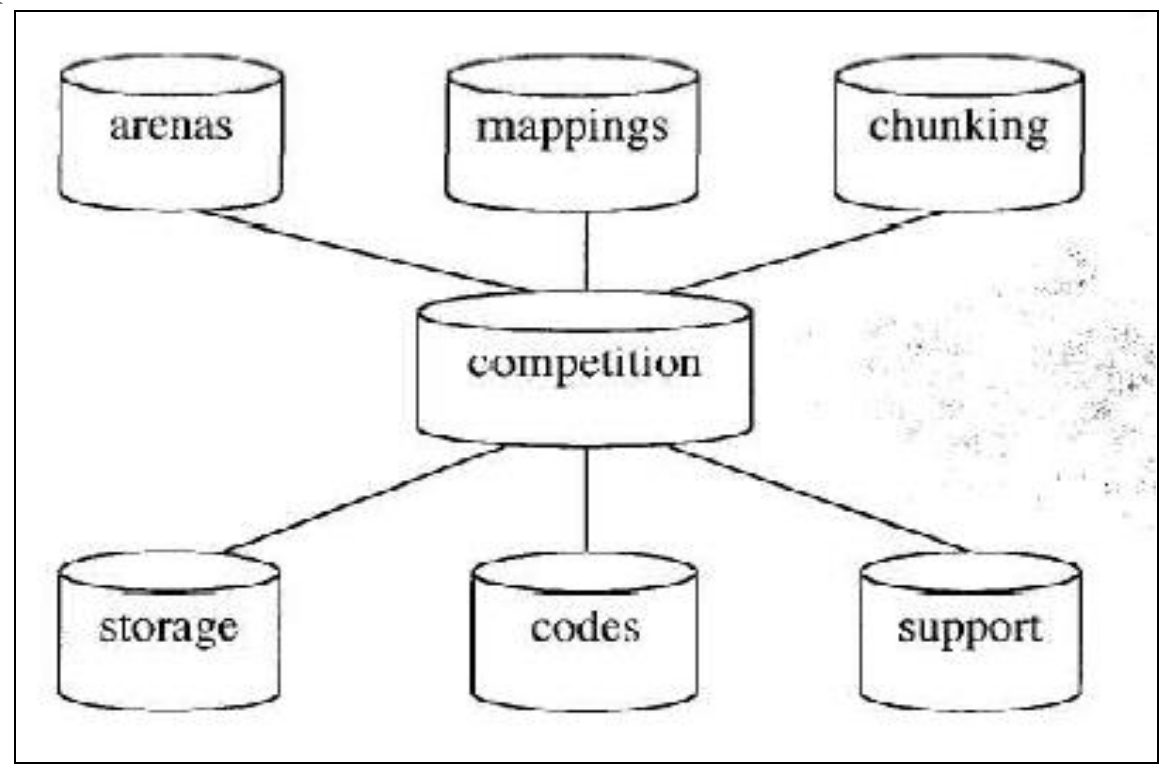

Fig.3: The Unified Competition Model. Taken from MacWhinney, 2005, p. 70. 
The components of the Unified Competition

Model (UCM) are briefly discussed below with the emphasis on the role of lexicon as the most crucial aspect in the competition.

1- Competition: In the classic version of the model, competition was based on cue summation and interactive activation. In the unified model, competition is based on resonance as well as activation summation.

2- Arenas: in UCM competition forms differ in each of the eight competition arenas. MacWhinney (2005) enumerates these arenas as follow:

In the auditory arena, competition involves the processing of cues to lexical forms based on both bottom-up features and activation from lexical forms. In the lexical arena, competition occurs within topological mapswhere words are organized by semantic and lexical type. In the morphosyntactic arena, there is an item-based competition between word orders and grammatical markings centered on valence relations. In the interpretive arena, there is a competition between fragments of mental models as the listener seeks to construct a unified mental model that can be encoded in long-term memory. In the arena of message formulation, there is a competition between communicative goals. Winning goals are typically initialized and topicalized. In the arena of expressive lexicalization, there is a competition between words for the packaging and conflation of chunks of messages. In the arena of sentence planning, there is a competition of phrases for initial position and a competition between arguments for attachment to slots generated by predicates. In the arena of articulatory planning, there is a competition between syllables for insertion into a rhythmic phrasal output pattern. (p. 73)

3- Cues and mappings: in productions forms compete to express underlying intentions or functions. In production functions or interpretations compete based on the cues from surface forms.

4- Chunking: UCM holds that learners control mappings at different levels of chunking or analysis. According to MacWhinney (1975, cited in MacWhinney, 2005) treated chunking levels in regard to three processes of rote (which involves the extraction of large chunks to be analyzed into later smaller chunks), analogy (which extracts new patterns based on the comparisons between stored chunks), and combination (based on the fact that existing chunks can be combined into larger proceduralized chunks). Chunking is one method for enhancing fluency, automatization and entrenchment (MacWhinney, 2006b)

5- Storage: learning new mappings depends on both shortterm and long-term memories. The operation of memory systems restrictsthe role of cue validity during both processing and acquisition.As an example,the process of subject-verb agreement for inverted word order in Italian is not fully learned until age eight, despite its high cue validity and high cue strength in adult speakers.

6- Codes: CM distinguishes between two components of the theory of code competition: theory of transfer (which accounts for the prediction of positive and negative transfer in different linguistic arenas) and the theory of codeinteraction (which accounts for code-selection, codeswitching, and code-mixing). According to MacWhinney (2005) in CM the selection of a certain code at a given moment during lexicalization depends on some factors like activation of previous lexical items, the impact of lexical gap, expressions of sociolinguistic choices, and conversational cues provided by the listener.

7- Resonance (cue support):this concept advocates the presentation of various types of input simultaneously. Resonance takes advantage of connection between arenas and representation forms to facilitate both processing and learning. Resonance puts emphasis on the fact that although various arenas are involved in different types of information they are not completely 'modularized' or 'encapsulated'.

McDonald and MacWhinney (1991) maintain that the order of cue acquisition by learners is determined by the validity of a cue in general in the language or the overall validity and they also introduce another type of validity named conflict validity defined as the validity of a cue in conflict situation which is a detriment of the strength with which cues are employed by adults with full mastery of language. They also define the process of cue learning as follow:

If there is a difference between the ranking of cues according to their overall validity and their conflict validity, full language mastery will involve shifting from initial weights that reflect overall validity to final weights that reflect conflict validity. (p. 408)

Sigalowitz and Lightbown (1999) maintain that in CM learning can happen without attention which means that learning is automatic(does not consume attentional capacity) and implicit (does not require intention) and that repetitive exposure to input is enough in order to learn. 
Ellis (1998) maintains that the information about frequencies, reliability, and validity of cues serves as the base of knowledge for production and comprehension of in lexicalist constraint satisfaction theories which assert that "sentence processing is the simultaneous satisfaction of the multiple probabilistic constraints afforded by the cues present in each particular sentence." (p.637)

\section{Contrastive Lexical competition}

Jordan (2004) maintains that the second language learners' main mission is to adjust the internal speechprocessing mechanisms from those proper in his/her L1 to those proper in the target language.

MacWhinney (2004a) emphasizes the role of CM in recovery from overgeneralization. He introduces three forces at play: analogic pressure, competition, and episodic encoding. It is claimed that L1 translations for L2 learners, reduces the load of competition between analogic pressure and episodic encoding. Taking the example of "kitty" from MacWhinney (2004a), it is argued that when simple translation easily works in obviating analogic pressure even before the introduction of any episodic encoding, L1-L2 comparison can be considered as a short-cut in language learning.

Ellis (2006) describes how in the case of multiple cues learners resort to Probabilistc Contrastive Model (PCM) based on which potential causes are assessed by contrasts computed in a 'focal set' defined as the contextually-determined set of events that the reasoned chooses to employ as input to the computation of that contrast.

The notorious notion of transfer has always been denounced as a negative trait resulting from the competition of L1 and L2. Tokowicz and MacWhinney (2005) maintain that the dominance of $\mathrm{L} 1$ in contrasting structural interpretations between the L1 and L2 is inevitable. They consider the competition between two languages as a more pervasive and persistent problem compared to transfer. What must be emphasized is the fact that the competition in CM must be viewed as the intermediate between form and function (MacWhinney, 1987) within which L1 forms can also be activated an cause interference. When L2 entry loses the competition constantly to its L1 counterpart L2 acquisition will slow down and even breaks off (Trsucott\& Smith, 2004). It can be claimed that in the Competition Model, the competition happens between: form and function, forms (cues) to a function, functions to a form and finally between L1 and L2. As an example in the case of competition between forms to be mapped onto a function and also the difference between two languages, it can be claimed that when subject-verb agreement is a weak cue in English (since the contrast in verb morphology to mark subject role does not always exist (I eat, you eat, they eat) (Su, 2001a; Su, 2001b), in Persian subject-verb agreement is a strong cue to the agent-patient relations in a sentence (man mixoram, to mixori, oumixorad) on the other hand subject-verb agreement is a highly valid and low-cost cue. In learning a language the most valid and the least costly cues of a language compete with less valid and the most costly ones, which finally dominate since there is more probability that they lead to better understanding (Thal\& Flores, 2001). Since subjects can be omitted in Persian without harming the meaning of a sentence, subject-verb agreement is a weak cue to the agent role in Persian. According to Rounds and Kanagy (1998) in L2 learning when the relative strength of the lexical semantic, morphological, and syntactic cues is different from their strength in the first language learners need to adjust the strength of various target language cues through developing comprehension strategies possibly different from those they employ in their L1.Pienemann's Processability Theory is in sharp contrast with $\mathrm{CM}$ in that it considers the concept of L1 transfer in CM as a bulk transfer and contends that L1 transfer is 'developmentally moderated' and occurs when the structure to be transferred is developmentally processable in the L2 system (Skehan, 2008).

What is crucial to bear in mind is the fact that the functions introduced in the $\mathrm{CM}$ resembles to that of Halliday's Systemic-Functional linguistics and deals with the form-meaning relations within the text and not in the real world. The argument is that through putting structures (mostly in the form of lexical units) into actual use and comparisons between L1 and L2 in conveying given functions, such problems as disagreement between languages inform-function mappings and the L1 interference through the transfer of its processing strategies (Su, 2001b) would be resolved. The studies in CM has been limited to (cross-linguistic) investigations in regard to the role of intrasentential cues such as word order, verb agreement, and case marking, noun animacy and so forth in determining the agent-patient relationship in simple declarative sentences (Su, 2004). Su (2004) emphasizes the role of discourse and context information in sentence processing. 
It is contended that Kroll, Michael, Tokowicz, and Dufour (2002) are misled when theycriticize the claim that "initial reliance on L1 gives way to independent access for L2 at the lexical Level" and also the claim that L1 must be considered as a "temporary crutch to enable L2 to bootstrap its way into the cognitive system" based on the argument that if a learners becomes a fluent bilingual they must be deprived from their $\mathrm{L} 1$ activities. What is reduced is not $\mathrm{L} 1$, rather the competition between L1 and L2 lexical units when employing each L1 or L2.

The argument is that the word association model (through which L2 words are dealt with through direct relation with their L1 translation equivalents) is the key in second language learning although it may gradually give way to concept mediation (which maintains that L2 words are directly connected to their meanings without any mediation by L1) as learner's proficiency develops.
To put into a Competition Model framework (figures 1 and 2) L1 translation equivalents to L2 lexicons help in passing valence bridges and settle competitions in comprehension and production.

Kroll and Stewart (1994, cited inKroll, Michael, Tokowicz, and Dufour, 2002) have proposed the revised hierarchical model in order to illuminate the implications of reliance on L1 at early stages for the form of word-toconcept connections. This model combines the word association and concept mediation alternatives into one single model in which the strength of the connection between words in L1 and L2 and concepts take on different values. The prior reliance on L1 to mediate access to meaning for L2 words is believed to establish robust connections from L2 to L1, but not the other way round. It is contended that the connection between words and concepts are stronger for L1 than for L2 (Kroll, Michael, Tokowicz, and Dufour, 2002).

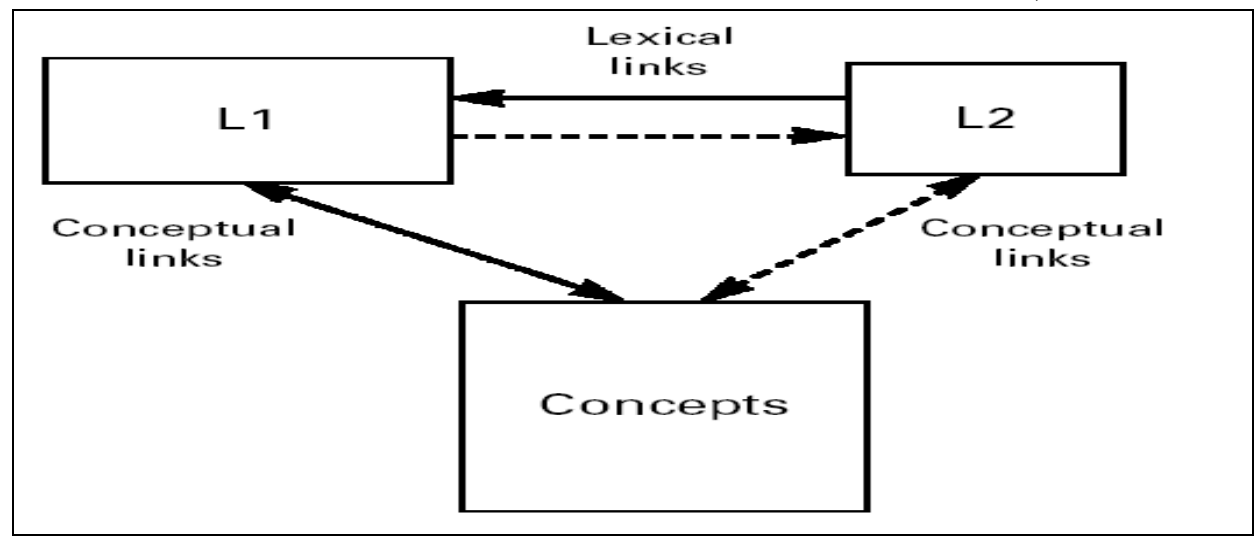

Fig.4: Revised hierarchical model. Taken from Kroll and Stewart, 1994, cited in Kroll, Michael, Tokowiczand Dufour, 2002, p.139

\section{Criticisms}

One criticism pertinent to $\mathrm{CM}$ is based on the notion of L1 transfer which is a reminiscent of Contrastive Studies. Pienemann's(cited in Skehan, 2008) idea of Processability theory calls into question CM based on the fact that L1 transfer in CM has been considered as a bulk transfer and that L1 transfer is 'developmentally moderated' and occurs when the structure to be transferred is developmentally processable in the L2 system (Skehan, 2008).

The second criticism can be set forth in regard to the fact that $\mathrm{CM}$ restricts form-function relations to textual analysis and does not investigate the effectof real language use. Its functionalism is bound to Halliday's Systemic
Functional Linguistics. The studies in CM has been limited to (cross-linguistic) investigations in regard to the role of intrasentential cues such as word order, verb agreement, and case marking, noun animacy and so forth in determining the agent-patient relationship in simple declarative sentences (Su, 2004).

\section{Final remarks}

The Competition Model can be considered as an attempt in bringing the notions of form and function together in an emergentist framework. CM believes in a lexicalist, bottom-up learning in its form-function mappings. What seems to be lacking within $\mathrm{CM}$ is a true allegiance to pragmatic and functional aspects of language forms which has been neglected to the advantage of studies 
investigating the functional aspects of structural units within the sentence rather than within the real contextual environment. The present CM, despite steering clear of Chomsky's theories, is bound to another formalist linguistic school i.e., structuralism in its form-function relations. A new Competition Model can be proposed which is more pragmatic-oriented through taking formulaic sequences as forms to be mapped onto real world pragmatic functions. CM must free itself from the mere focus on sentence processing studies and involve itself with more pragmatic manifestations of form-function relations. It is claimed that within the models in which there is an architecture that utilizes lexical categories to build "valence bridges", L1-L2 translation equivalents facilitate crossing valence bridges which helps in discovering forthcoming elements and filling syntactic slots. Based on the revised hierarchical model, it seems more reasonable to see to L1-L2 contrast as a facilitator of lexical links through which L1 lexical translation equivalents facilitate $\mathrm{L} 2$ acquisition, rather than as a competition which hinders L2 learning because of interference and transfer.

\section{REFERENCES}

[1] Behrens, H. (2009). Grammatical categories. In E. L. Bavin (Ed.), The Cambridge handbook of child language. Cambridge, UK: Cambridge University Press.

[2] Dopke, S. (2000). Generation of and retraction from crosslinguistically motivated structures in bilingual first language acquisition. Bilingualism: Language and Cognition, 3(3), 209-226.

[3] Ellis, N. C. (1998). Emergentism, connectionism and language learning. Language Learning, 48(4), 631-664.

[4] Ellis, N. C. (1999). Cognitive approaches to SLA. Annual Review of Applied Linguistics, 19, 22-42.

[5] Ellis, N. C. (2002). Frequency effects in language processing: A review with implications for theories of implicit and explicit language acquisition. SSLA, 24, 143188.

[6] Ellis, N. C. (2006). Selective attention and transfer phenomena in L2 acquisition: Contingency, cue competition, salience, interference, overshadowing, blocking and perceptual learning. Applied Linguistics, 27(2), 164-194.

[7] Harrington, M., \& Dennis, S. (2002). Input-driven language learning. SSLA, 24, 261-268.

[8] Jordan, G. (2004). Theory construction in second language acquisition. Amterdam, Netherlands: John Benjamins Publishing Co.

[9] Kempe, V., \&MacWhinney, B. (1998). The acquisition of case making by adult learners of Russian and German. SSLA, $20,543-587$.
[10] Kroll, J. F., Miachael, E., Tokowicz, N., \& Dufour, R. (2002). The development of lexical fluency in a second language. Second Language Research, 18(2), 137-171.

[11] MacWhinney, B. (1987). Applying the competition model to bilingualism. Applied Psycholinguistics, 8, 315-327.

[12] MacWhinney, B. (1994). The dinosaurs and the ring. In R. Corrigan, G. Iverson \& S. Lima (Eds.), The reality of linguistic rules. Amsterdam, Netherlamd: Benjamins.

[13] MacWhinney, B. (2004a). A multiple process solution to the logical problem of language acquisition. Journal of Child Language, 31, 883-914.

[14] MacWhinney, B. (2004b). Parameters or cues. Bilingualism: Language and Cognition, 7(1), 35-36.

[15] MacWhinney, B. (2005). Extending the competition model. International Journal of Bilingualism, 9(7), 69-84.

[16] MacWhinney, B. (2006a). Emergent fossilization. In Z. Han \& T. Odlin (Eds.), Studies of fossilization in second language acquisition (pp. 134-156). Clevedon, England: Multilingual Matters.

[17] MacWhinney, B. (2006b). Emergentism: Use often and with care. Applied Linguistics, 27(4), 729-740.

[18] MacWhinney, B., \&Pleh, C. (1997). Double agreement: Role identification in Hungarian. Language and Cognitive Processes, 12(1), 67-102.

[19] MacWhinney, B., Bates, E., \&Kliegl, R. (1984). Cue validity and sentence interpretation in English, German, and Italian. Journal of Verbal Learning and Verbal Behavior, 23, 127150.

[20] MacWhinney, B., Pleh, C., \& Bates, E. (1985). The development of sentence interpretation in Hungarian. Cognitive Psychology, 17, 178-209.

[21] MacWhinney, B., Taraban, R., \& McDonald, J. (1989). Language learning: Cues or rules? Journal of Memory and Language, 28, 255-277.

[22] Matessa, M., \& Anderson, J. R. (2000). Modelling focused learning in role assignment. Language and Cognitive Processes, 15(3), 263-292.

[23] McDonald, J. L., \&MacWhinney, B. (1991). Levels of learning: A comparison of concept formation and language acquisition. Journal of Memory and Language, 30, 407-430.

[24] Rounds, P. L., \&Kanagy, R. (1998). Acquiring linguistic cues to identify agent: Evidence from children learning Japanese as a second language. SSLA, 20, 509-542.

[25] Segalowitz, N., \&Lightbown, P. M. (1999). Psycholinguistic approaches to SLA. Annual Review of Applied Linguistics, 19, 43-63.

[26] Skehan, P. (2008). Interlanguage and language transfer. In B. Spolsky\& F. M. Hult (Eds.), The handbook of educational linguistics (pp. 412-423). Malden, MA: Blackwell Publishing. 
[27] Su, I-R. (2001a). Context effects on sentence processing: A study based on the competition model. Applied Psycholinguistics, 22, 167-189.

[28] Su, I-R. (2001b). Transfer of sentence processing strategies: A comparison of L2 learners of Chinese and English. Applied Psycholinguistics, 22, 83-112.

[29] Su, I-R. (2004). The effects of discourse processing with regard to syntactic and semantic cues: A competition model study. Applied Psycholinguistics, 25, 587-601.

[30] Thal, D. J., \& Flores, M. (2001). Development of sentence interpretation strategies by typically developing and latetalking toddlers. Journal of Child Language, 28, 173-193.

[31] Tokowicz, N., \&MacWhinney, B. (2005). Implicit and explicit measures of sensitivity to violations in second language grammar. SSLA, 27, 173-204.

[32] Truscott, J., \& Smith, M. S. (2004). Acquisition by processing: A modular perspective on language development. Bilingualism: Language and Cognition, 7(1), $1-20$. 